\title{
O PESSEGUEIRO NO SISTEMA DE POMAR COMPACTO: VII. COMPORTAMENTO DE NOVAS SELEÇÕES IAC SOB PODA DRÁSTICA BIENAL ${ }^{1}$
}

\author{
W. BARBOSÁ' ; F.A.CAMPO-DALL'ORTO'; M. OJIMA; A.A. LOVATE \\ Instituto Agronómico de Campinas, C.P. 28, 13001-970 Campinas, SP. \\ R.R. dos SANTOS \\ Estação Experimental de Monte Alegre do Sul/IAC, C.P. 50, CEP: 13910-000 - Monte Alegre do Sul,SP.
}

\begin{abstract}
RESUMO: Pesquisou-se durante seis anos o comportamento reprodutivo de cinco novas seleçzes de pêssego e

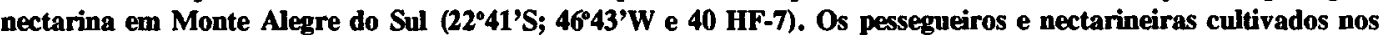
espaçamentos de $4 \times 0,5 \mathrm{~m}$ e $4 \times 1 \mathrm{~m}$, equivalentes a 5000 e 2500 plantas/ha, respectivamente, foram podados drasticamente, a cada dois anos, apos a colheita. Em ambos espaçamentos,'Aurora-2' e IAC 282-24 apresentaram as melhores produções acima de 19 toneladas/ha. Verificou-se nos ciclos subseqüentes a cada poda da copa, tendência de menor produçâo das plantas, principalmente em material de ciclo mediano. No segundo ano ap6s a poda drástica, os pessegueiros e nectarineiras produxiram normalmente. No espaçamento de $4 \times \mathbf{0 , 5}$, as plantas apresentaram acréscimo médio de $12 \%$ na produção, em relação a $4 \times 1 \mathrm{~m}$, diminuindo porém, o peso do produto final. Os maiores frutos com peso médio acima de $100 \mathrm{~g}$ foram produzidos pelas seleçōes IAC 6782-83 e IAC 282-24.

Descritores: péssego, nectarina, Prunus persica; seleções IAC; pomar compacto; poda drástica; produtividade.
\end{abstract}

\section{THE PEACH MEADOW ORCHARD SYSTEM: VII. PERFORMANCE OF NEW IAC SELECTIONS ON BIENNAL DRASTIC PRUNING}

\begin{abstract}
ARSTRACT: Five new peach and nectarin selections were grown under the meadow orchard system, with bienal drastic pruning, at $4 \mathrm{~m} \times 0.5 \mathrm{~m}$ and $4 \mathrm{~m} \times 1 \mathrm{~m}$ spacings. The experimental area was located in Monte Alegre do Sul, $\left(22^{\circ} 41^{\prime} \mathrm{S} ; 46^{\circ} 43^{\prime} \mathrm{W}\right.$ and 40 hours below $7^{\circ} \mathrm{C}$ ) State of São Paulo, Brazil. Results obtained during the six years of experiments clearly indicate that the 'Aurora-2' and IAC 282-24 peach selections were the best, with an average yield above 19 ton per hectare. For $4 \mathrm{~m} \times \mathbf{0 . 5 m}$ spacing, with a density of 5000 plants per hectare, the productivity increased about $12 \%$, however decreasing the weight of the final product. The best average weights of the fruits, above of $100 \mathrm{~g}$, were obtained by the IAC $6782-83$ and IAC $282-24$ selections.
\end{abstract}

Keg Words: peach, nectarine, Prunus persica, IAC selections, meadow orchard, drastic pruning, productivity.

\section{INTRODUÇÃO}

$O$ cultivo de pessegueiros em altas densidades populacionais vem sendo pesquisado $e$ aperfeiçoado desde a década de 70 . Ao longo dos anos conseguiu-se desenvolver tecnologia para o plantio de até 10.000 plantas/ha, em diversos espaçamentos. Com isso, os objetivos perseguidos foram sendo paulatinamente atingidos, como a maior produção possível por área, logo nos primeiros anos de cultivo e com redução de mão de obra especializada (LORETI et al., 1990; EREZ, 1976; 1985; CAMPO DALL'ORTO et al., 1984; BARGIONI et al., 1983; COUVILLON, 1985; LORETI \& PISANI, 1990; BARBOSA et al., 1990a).

Os principais tópicos pesquisados sobre o assunto, em diversos países, em cerca de cinquenta trabalhos científicos, têm sido: métodos de propagação, porta-enxertos ananicantes de copa, espaçamentos de plantio, tipos e épocas de poda, manejo de plantas e de frutos e seleção de

\footnotetext{
${ }^{1}$ Pesquisa integrante do projeto IAC: "Pessegueiro: Melhoramento Genético e Cultural".

${ }^{2}$ Bolsista do CNPq.
}

Sci. agric., Piracicaba, 51(1):94-98, jan./abr., 1994 
cultivares e de climas apropriados (EREZ \& YABLOWTTZ, 1991; CAMPO DALL'ORTO et al., 1992; GUERREIRO et al., 1980; 1988; CHALMERS et al., 1978; LORETI et al.,1990, BARBOSA et al., 1989; 1990a; 1990b; 1990c; 1991a; 1991b; 1992). Acredita-se que, entre os temas abordados, a seleção de cultivares que apresentem rusticidade vegetativa e reprodutiva constitui o de maior relevância, pois nem todo material se adapta ao sistema. No cultivo de pessegueiros em ultra densidade, por exemplo, com poda drástica anual, os cultivares têm de ser especificamente precoces e rústicos, para que propiciem desenvolvimento rápido da copa, e, em média, diferenciem gemas floríferas no 50 ,floresçam no 9o e amadureçam no os frutos no $12 \underline{\underline{o}}$ mês da poda drástica pós colheita. Somente dessa forma, as plantas conseguem repetir igualmente $o$ ciclo no ano seguinte e com produções satisfatórias. Quando se empregam cultivares medianos e tardios, em pomares compactos com poda drástica anual, verifica-se defíciência no ciclo de desenvolvimento vegetativo e no processo de diferenciação floral, diminuindo acentuadamente a frutificação dos pessegueiros (BARBOSA,1989). Além disso, em regiões subtropicais há necessidade da seleção de cultivares próprios, bem adaptados à ecologia local, pois os cultivares introduzidos de países frios freqüentemente tornam-se improdutivos devido à falta de adaptação climática (BARBOSA et al., 1990c; 1993).

Neste contexto, o Instituto Agronômico de Campinas vem desenvolvendo trabalhos de melhoramento genético e cultural, visando as seleções de pêssegos e nectarinas de alta qualidade organoléptica, e, principalmente, com rusticidade aos cultivos adensados. São apresentados no trabalho, o comportamento de cinco novas seleções e sua viabilidade ao cultivo em pomar compacto, com poda drástica bienal pós colheita.

\section{MATERIAL E METODOS}

Utilizaram-se na experimentação pessegueiros e nectarineiras cultivados na Estação Experimental de Monte Alegre do Sul (22041'S.; $46^{\circ} 43^{\prime} \mathrm{W}$. e $40 \mathrm{HF}-7$ ), do IAC. As seleções de pêssegos - 'Aurora-2', IAC 282-24, IAC 6782-83, e IAC 280-28, e de nectarina- IAC N 2680-91, foram plantadas em dois lotes contíguos, nos espaçamentos de $4 \mathrm{~m} \times 0,5 \mathrm{~m}$ (5000 plantas/ha) e $4 \mathrm{~m} \times 1 \mathrm{~m}$ (2500 plantas/ha) As podas drásticas das copas, a $50 \mathrm{~cm}$ do solo, foram efetuadas a cada dois anos, imediatamente após a colheita dos frutos. Para cada material e espaçamento utilizaram-se dez plantas, que, com exceção da poda drástica, receberam os tratos culturais rotineiros, como: pincelamento do tronco, desbrota, capina, pulverizações fitossanitárias, irrigação, adubação e quebra de endodormência. Para facilitar o manejo, as plantas eram rebaixadas, anualmente, no inverno, a cerca de $2,5 \mathrm{~m}$ de altura. No raleio, deixaram-se um e dois frutos por ramo médio $e$ vigoroso, respectivamente. Durante os seis anos de pesquisa, os frutos foram colhidos, contados e pesados, sempre de fins de setembro a meados de novembro, obedecendo a época normal de maturação de cada material. Aos dados de produção e peso médio dos frutos calcularam-se os intervalos de confiança para a média, ao nível de 95\%.

\section{RESULTADOS E DISCUSSÀO}

Os pessegueiros e as nectarineiras iniciaram a rebrota dos troncos cerca de quinze dias após a decepa da copa. Verificaram-se entre as seleções pesquisadas, diferenças na quantidade de brotos emitidos e no desenvolvimento dos ramos jovens. As plantas de 'Aurora-2' e IAC 282-24 foram as mais rústicas na brotação, que se desenvolveu de forma bem vigorosa. Nessas seleções, as três e cinco pernadas deixadas por tronco, respectivamente, nos espaçamentos de $4 \mathrm{~m} \times 0,5 \mathrm{~m}$ e $4 \mathrm{~m} \times 1 \mathrm{~m}$, apresentaram profusa emissão de ramos laterais, responsáveis pela frutificação. As seleções 'IAC 6782-83', 'IAC 280-28'e IAC N 2680-91, por sua vez, tiveram desenvolvimento vegetativo mais lento, e principalmente, com menor emissão de ramos laterais.

Ressalte-se que, as melhores florações e frutificações efetivas ocorreram, porém, no segundo ano de desenvolvimento pós poda, pois as copas apresentaram-se naturalmente maiores, apesar de produzirem ramos internos mais finos $e$ improdutivos. Essa constatação, bem evidente em material de ciclo mediano, pode encontrar explicação no processo de diferenciação floral das gemas. Com podas drásticas mais tardias, realizadas após o final de outubro, ocorre certo atraso no desenvolvimento dos brotos, e por conseqüente, na época de indução floral, prejudicando a quantidade de gemas floríferas das plantas (BARBOSA, 1989). No segundo ciclo vegetativo, em que não ocorre a poda drástica da copa, as gemas florais se diferenciam em época 
normal (janeiro), proporcionando elevação no número de flores $\mathrm{e}$ de frutos nos ramos (BARBOSA et al., 1990b).

Em ambos espaçamentos, o 'Aurora2'apresentou-se como um dos mais produtivos, apesar de ser material considerado mediano: ciclo florada-maturação de 120 dias. Em trabalhos anteriores de poda drástica anual pós-colheita, realizados na Estação Experimental de Jundiaí

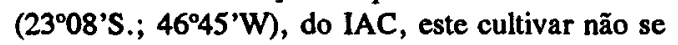
desenvolveu adequadamente, devido às colheitas $e$ consequentes podas tardias (BARBOSA, 1989). Convém destacar que, quando se realiza anualmente a referida poda, há necessidade de se trabalhar com cultivares bem precoces, a exemplo de 'Tropical' e 'Flordaprince', que apresentam ciclo de maturação dos frutos de aproximadamente 80 dias. As colheitas sendo precoces permitem a realização das podas mais cedo, entre meados de setembro a início de outubro, em região subtropical (EREZ, 1985; BARBOSA et al., 1991b). Ao se utilizar o 'Aurora-2' em pomares bem compactos, há, então, necessidade de lançar mão das podas drásticas bienais. Desta forma, suas plantas não sofrem interrupção de crescimento a cada 12 meses, permitindo colheitas satisfatórias ao longo dos anos. Destaque-se que, mesmo utilizando este processo, o 'Aurora-2' ainda apresentou tendência de menor frutificação nos ciclos subsequentes a cada poda, fato que não comprometeu a produtividade acumulada em seis anos de controle.

O IAC 282-24, de ciclo mais precoce, comportou-se de forma diferenciada ao 'Aurora-2', florescendo e frutificando uniformemente todos os anos. As colheitas e podas realizadas precocemente devem ter possibilitado, a esta seleção, indução e diferenciação floral em épocas mais adequadas, ocorrendo menor variabilidade no volume de floração e na porcentagem de frutificação efetiva.

As maiores produções ocorreram no espaçamento menor, equivalente a 5000 indivíduos por hectare, (figura 1). Nesta densidade populacional, os cultivares e seleções superaram a produção obtida no lote de 2500 plantas/ha, verificando-se aumento médio de $12 \%$ na produtividade (figura 2). Estes dados confirmam os resultados relatados por CAMPO DALL'ORTO et al.,(1984), quando obtiveram aumento de produtividade de cerca de $8 \%$, comparando 29 seleções nas densidades populacionais de 11.428 e 6.666 plantas/ha, em espaçamentos de $3 \times 0,5 \times 0,5 \mathrm{~m}$ (renque duplo) e $3 \times 0,5 \mathrm{~m}$ (renque simples) respectivamente. Resultados similares são descritos, também, por outros autores quando aumentaram a densidade de plantio de pessegueiros e nectarineiras (LORETI \& PISANI, 1990; GUERREIRO et al., 1980; RECUPERO et al., 1985). Apesar da frutificação individual ser menor nas altas densidades populacionais, a produtividade, sem dúvida, torna-se mais elevada devido ao aumento do número de plantas por área. Ao se desejar ainda, maior produtividade, há tendência em diminuir a intensidade de raleio, mantendo-se mais frutos na planta. Com isto, o peso médio final do produto pode decrescer acentuadamente, comprometendo a comercialização (BARBOSA et al., 1991a).

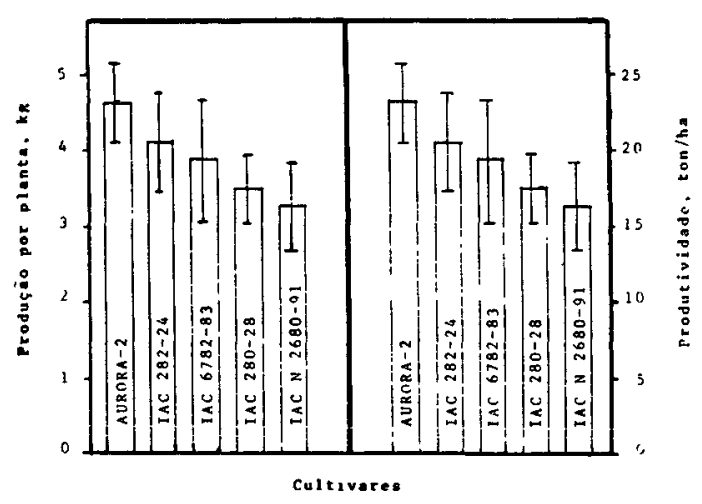

Figura 1 - Produção média por planta (A) e produtividade (B) de pessegueiros e nectarineiras IAC cultivados por 6 anos no espaçamento de $4 \times 0,5 \mathrm{~m}$, em pomar compacto, sob poda drástica bienal. As linhas verticais sobre colunas indicam os intervalos de confiança para a média ao nível de $95 \%$.

Os pêssegos 'Aurora-2' e IAC 282-24, apesar de superarem os demais em produtividade, não apresentaram os maiores frutos em termos de peso médio. Das seleções pesquisadas, somente a IAC 6782-83 obteve, em média, frutos acima de $120 \mathrm{~g} \mathrm{em}$ ambos espaçamentos (figura 3 ).

No espaçamento de $4 \times 0,5 \mathrm{~m}$ ocorreu diminuição de peso médio dos frutos em cerca de $10 \%$. A maioria do material, no entanto, apresentou adequado peso médio final, superior a $80 \mathrm{~g} /$ fruto, mostrando, assim, potencial para esta característica (figura 3). 


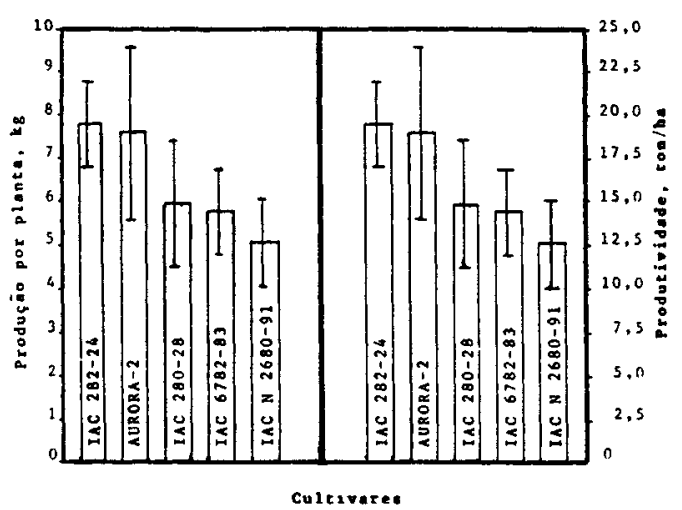

Figura 2 - Produção média por planta (A) e produtividade (B) de pessegueiros e nectarineiras IAC cultivados por 6 anos no espaçamento de $4 \times 1,0 \mathrm{~m}$, em pomar compacto, sob poda drástica bienal. As linhas verticais sobre colunas indicam os intervalos de confiança para a média ao nível de $95 \%$.

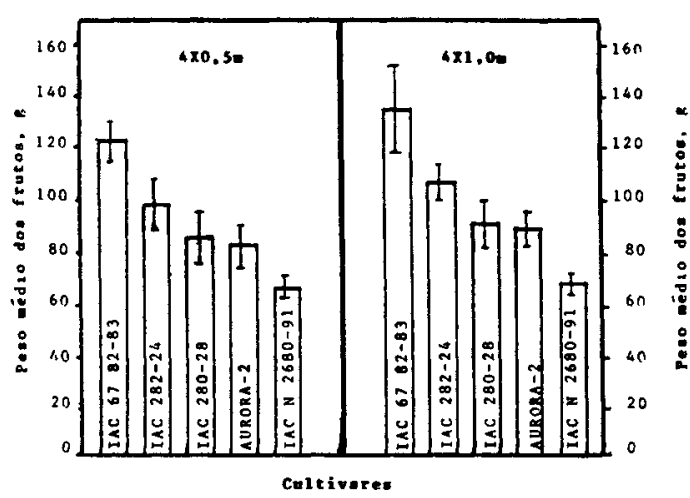

Figura 3 - Peso médio de pêssegos e nectarinas IAC cultivados por 6 anos nos espaçamentos de 4 × 0,5 (A) e 4 × 1,0 m (B), em pomar compacto, sob poda drástica bienal. As linhas verticais sobre colunas indicam os intervalos de confiança para a média ao nível de $95 \%$.

\section{CONCLUSÕES}

1. De cinco seleções IAC pesquisadas, 'Aurora-2' e IAC 282-24 apresentaram-se as mais produtivas, quando cultivadas no sistema de pomar compacto com poda drástica bienal.
2. O 'Aurora-2' e IAC 282-24 produziram, em média, acima de 19 t/ha.ano, nos espaçamentos de $4 \times 0,5 \mathrm{~m}$ e $4 \times 1 \mathrm{~m}$.

3. Os maiores frutos com peso médio acima de $100 \mathrm{~g}$ foram produzidos pelas seleções IAC 6782-83 e IAC 282-24, no espaçamento de $4 \times 1 \mathrm{~m}$.

\section{AGRADECIMENTO}

Os autores agradecem ao Auxiliar de Campo, LÁZARO DE GODÓI às colheitas controladas $\mathrm{e}$ os tratos culturais dos pessegueiros $\mathrm{e}$ nectarineiras do ensaio.

\section{REFERÊNCIAS BIBLIOGRÁFICAS}

BARBOSA, W. Desenvolvimento vegetativo e reprodutivo do pessegueiro em pomar compacto sob poda drástica anual. Piracicaba, 1989. 154p. Dissertação (Mestrado) - Escola Superior de Agricultura "Luiz de Queiroz", Universidade de São Paulo).

BARBOSA, W.; CAMPO-DALL'ORTO, F.A.; OJMA, M. O pessegueiro no sistema de pomar compacto: Conjeturas, experimentação e prática. $O$ Agronómico, Campinas, v.41, n.1, p.26-39, 1989.

BARBOSA, W.; CAMPO-DALL'ORTO, F.A.; OJMA, M. O pessegueiro no sistema de pomar compacto: $V$. Pesquisas do Instituto Agronômico na década de 80.0 Agronomico, Campinas, v.42, n.1, p.35-44, 1990a.

BARBOSA, W.; CAMPO-DALL'ORTO, F.A.; OJMA, M.; SAMPAIO, V.R. O pessegueiro no sistema de pomar compacto: III. Épocas de poda drástica na diferenciação floral. Bragantia, Campinas, v.49, n.1, p.147-155, $1990 \mathrm{~b}$.

BARBOSA, W.; CAMPO-DALL'ORTO, F.A.; OIIMA, M; SAMPAIO, V.R.; BANDEL, G. Ecofisiologia do desenvolvimento regetativo e reprodutivo do pessegueiro em regiano subtropical. Campinas: Instituto Agronômico, 1990. 37p. (LAC. Documentos, 17)

BARBOSA, W.; CAMPO-DALL'ORTO, F.A.; OIMA, M.; MARTINS, F.P.; IGUE, T. O pessegueiro no sistema de pomar compacto: IV. Intensidade e época de raleio dos frutos dos cultivares 'Tropical' e 'Aurora-1'. Bragantia, Campinas, v.50, n.1, p.93102, 1991 a.

BARBOSA, W.;OJIMA, M; CAMPO DALL'ORTO, F.A.; SAMPAIO, V.R.; MARTINS, F.P.; IGUE, T. O pessegueiro no sistema de pomar compacto: $\Pi$. Influência no desenvolvimento das plantas. Campinas: Instituto Agronômico de Campinas, 1991b. 26p. (IAC. Boletim Científico, 22). 
BARBOSA, W.; CAMPO-DALL'ORTO, F.A.; OJMA, M.; SANTOS, R.R. O pessegueiro no sistema de pomar compacto: VI. Frutificação efetiva e raleio químico em seleções IAC. Bragantia, Campinas, v.51, n.1, p.63-67, 1992.

BARBOSA, W.; OJMA, M.; CAMPO DALL'ORTO, F.A.; RIGITANO, O.; MARTINS, F.P.; SANTOS, R.R.; CASTRO, J.L. Melhoramento do pessegueiro para regiōes de clima subtropical-temperado: realizaçōes do Instituto Agronomico no período de 1950-1990. Campinas, Instituto Agronômico, 1993. 30p. Datilografado.

BARGIONI, G.; LORETI, F.; PISANI, P.L. Performance of peach and nectarine in a high density system in Italy. HortScience, Alexandria, v.18, n.2, p.143-146, 1983.

CAMPO DALL'ORTO, F.A.; OJMA, M.; BARBOSA, W.; MARTINS, F.P. O nanismo do pessegueiro induzido pela enxertia no damasqueiro japonês. Pesquisa Agropecuária Brasileira, Brasîia, v.27, n.3, p.517-521, 1992.

CAMPO-DALL'ORTO, F.A.; OJMM, M.; BARBOSA, W.; TOMBOLATO, F.A.C.; RIGITANO, O.; ALVES, S. Cultivo de pessegueiros precoces no sistema de pomar compacto com poda drástica anual de renovação da copa. Pesquisa Agropecuária Brasileira, Brasilia, v.19, n.6, p.719-727, 1984.

COUVILLON, G.A. Propagation and performance of inexpensive peach trees from cuttings for high density peach plantings. Acta Horticulturae, Wageningen, v.173, p.271-281, 1985 .

CHALMERS, D.; ENDE, van den B.; HEEK, van L. Productivity and mechanization of the Tatura trellis orchard. HortScience, Alexandria, v.13, n.5, p.517$521,1978$.

Sci. agric., Piracicaba, 51(1):94-98, jan./abr., 1994
EREZ, A. Meadow orchard for the peach. Scientia Horticulturae, Amsterdam, v.5, p.43-48, 1976.

EREZ, A. Peach meadow orchards. Acta Horticulturae, Wageningen, v.173, p.405-411, 1985.

EREZ, A.; YABLOWTTZ, Z. Rooting of peach hardwood cuttings for the meadow orchard. Scientia Horticulturae, Amsterdam, v.15, p.137-144, 1991.

GUERREIRO, R.; LORETI. F.; MASSAI, R. Evaluation of new peach roostocks for high-density planting systems. HortScience, Alexandria, v.23, n.1, p.117-118, 1988.

GUERREIRO, R.; LORETI, F.; NATALI, S. Eight years of observations on a peach double-row planted orchard. Acta Horticulturae, v.114, p.362-363, 1980.

LORETI, F.; PISANI, P.L. Elevate densitè di piantagione e relative forme di allevantamento. Atti convegno "La potatura degli alberi da frutto negli anni 90". Verona, v.27, apile, p.39-68, 1990.

LORETI, F.; MASSAI, R.; MORINI, S. Effect of training system, planting density and rootstock on growth and quality of peach shoots. Advances in Horticultural Science, New York, v.5, p.45-47, 1991.

RECUPERO, S.; MONASTRA, F.; DAMIANO, C. Study on planting densities of peach and nectarine cultivars on diferent rootstocks. Acta Horticulturae, Wageningen, v.173, p.311-322, 1985.

Enviado para publicação em 26.07 .93

Aceito para publicação em 12.08 .93 